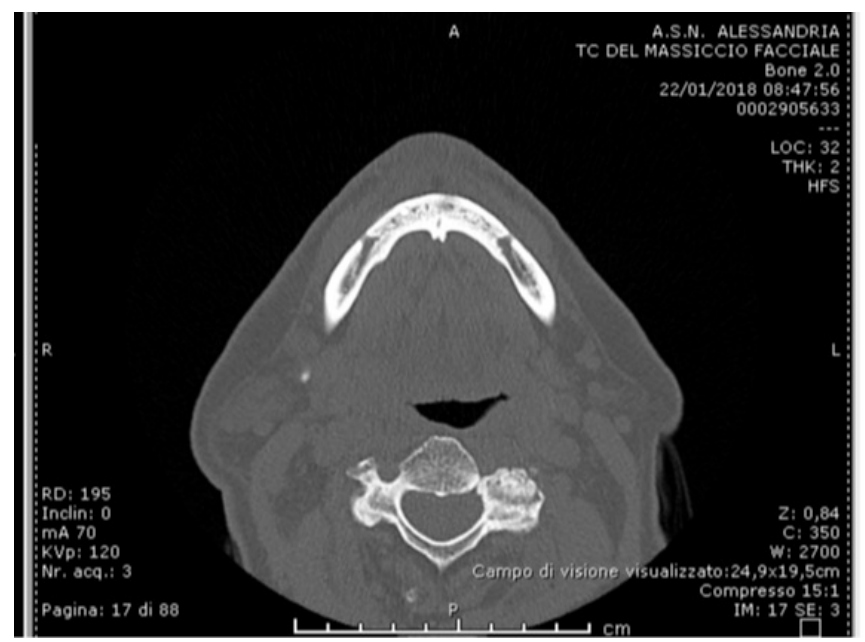

\title{
OSTEONECROSIS OF THE JAW (ONJ) IN DIFFERENTIATED THYROID CANCER (DTC): A CASE REPORT AFTER ZOLEDRONIC ACID AND LENVATINIB TREATMENT
}

\begin{abstract}
Maura Rossi ${ }^{1}$, Antonella Fasciolo ${ }^{1}$, Paola giorgia Brigo ${ }^{1}$, Giulia Piacentini ${ }^{1}$, Fulvia Blengio ${ }^{1}$, Carmela Riccio ${ }^{1}$, Pamela Francesca Guglielmini ${ }^{1}$, Silvia Zai ${ }^{1}$, Elena Traverso ${ }^{1}$, Riccardo Alberto Campora ${ }^{1}$, Roberto Manfredi ${ }^{1}$, Vittorio Fusco
\end{abstract}

1 Azienda Ospedaliera SS. Antonio e Biagio e Cesare Arrigo

Funding: The author(s) received no specific funding for this work.

Potential competing interests: The author(s) declared that no potential competing interests exist.

\section{Abstract}

Patients with Differentiated Thyroid Cancer (DTC) and bone metastases (BM) have generally poor prognosis and low survival rate. The management is based on radioiodine therapy, local procedures and systemic therapies, such as kinase inhibitors. The use of antiresorptive drugs (bisphosphonates or denosumab) is essential to prevent skeletalrelated events (SREs), and improve quality of life. The concomitant association of antiangiogenic therapies and antiresorptive treatments have been associated with occurrence of Osteonecrosis of the Jaw (ONJ). We report a case of ONJ in a 74 years old man treated with concomitant lenvatinib and zoledronic acid for advanced iodine-refractory DTC and BM, developing ONJ almost 8 years after start of zoledronic acid and 34 months after beginning of lenvatinib.

\section{Background:}

Distant metastases are detected in $10 \%-15 \%$ of patients diagnosed with DTC. Bone metastases (BM) are rare with an incidence of about $5.5 \%$ and are generally associated with poor prognosis and low survival rates ${ }^{[1][2]}$. BM from thyroid 
carcinoma may also cause severe complications. Multidisciplinary and personalized management is required to achieve disease control and prevent skeletal-related events (SREs), including bone fracture, spinal cord compression and hypercalcemia. The treatment is based on radioiodine therapy, local procedures (surgery, radiotherapy, percutaneous techniques) and systemic therapies, such as kinase inhibitors (lenvatinib) and antiresorptive drugs (bisphosphonates or denosumab) ${ }^{[3][4]}$. There are limited data to potential efficacy of concomitant use of antiresorptive agents and kinase inhibitors in BM of DTC and the optimal dosing interval for bone-directed therapy is still uncertain. Antiangiogenic therapies as well as antiresorptive treatments have been associated with occurrence of Osteonecrosis of the Jaw (ONJ), a rare but potentially serious adverse event. Lenvatinib, an oral inhibitor of vascular endothelial growth factor receptors 1,2, and 3, fibroblast growth factor receptors 1 through 4, platelet-derived growth factor receptor $\alpha$, RET, and KIT, showed good clinical activity in a phase 2 study; in a phase III trial, as compared with placebo, was associated with significant improvements in progression-free survival[ ${ }^{[5]}$. ONJ associated to lenvatinib has been described in a patient with Hurthle cell thyroid cancer ${ }^{[6]}$.

\section{Materials and Methods:}

We report a case of ONJ in 74 years old male patient treated with concomitant lenvatinib and zoledronic acid for advanced iodine-refractory DTC and BM.

\section{$\underline{\text { Results }}$}

In December 2005, a 59 years old man presented with lung, lymph nodes and bone metastases. He had a history of multiple comorbidities, including adrenal insufficiency in steroid replacement therapy. The patient underwent total thyroidectomy and lymph node dissection. Pathological examination revealed tall cell variant of papillary thyroid carcinoma (PTC). This variant is more aggressive than classic PTC with a higher recurrence rate and shorter survival. The patient received treatment with thyroid hormone replacement therapy and radioactive iodine-131(RAl) therapy.

Futhermore, on November 2007 he started a monthly treatment with intravenous 4 mg zoledronic acid. At that time and in 2008 dental panoramic Radiography showed presence of implants without severe bone alterations (Fig 1). 

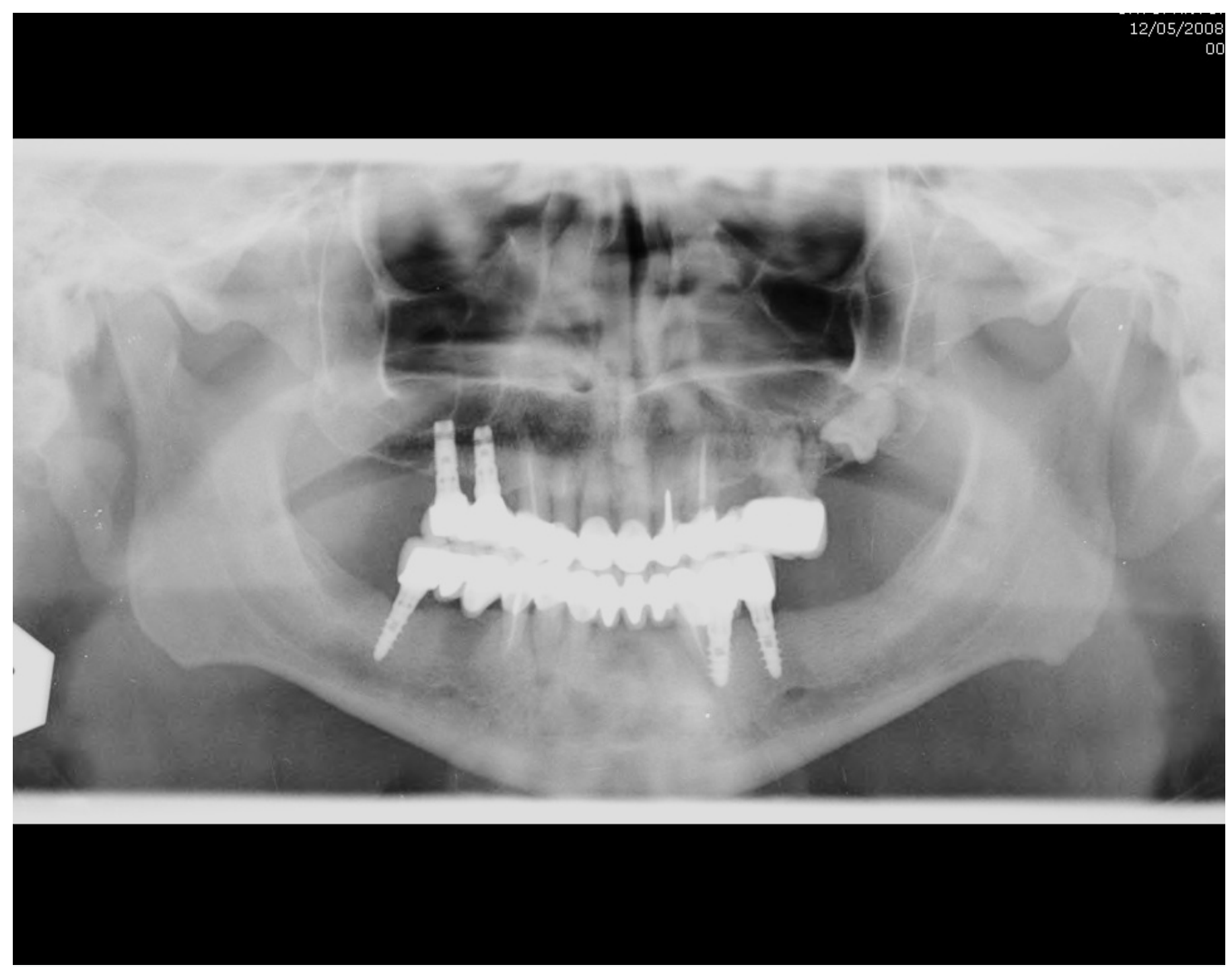

Fig 1. Dental panoramic radiography at May 2008

In October 2012, the disease became iodine-refractory. The patient started treatment with lenvatinib, a multikinase inhibitor with antiangiogenic activity, approved for the treatment of adult patients with progressive, locally advanced or metastatic, differentiated thyroid carcinoma that is refractory to radioactive iodine.

In August 2015 he underwent a visit at the maxillofacial unit (Osteonecrosis of the jaw prevention and screening office) due to gengival retractions and pain (both at left maxilla and at mandible). No bone exposure was evident, and a "stage 0" disease (according to AAOMS recommendations and staging system) was suspected. A CT scan revealed extensive bone alterations at right and left parts of mandible (both osteolysis and ostesclerosis, with several areas of sequestrum) (Fig 2) . 


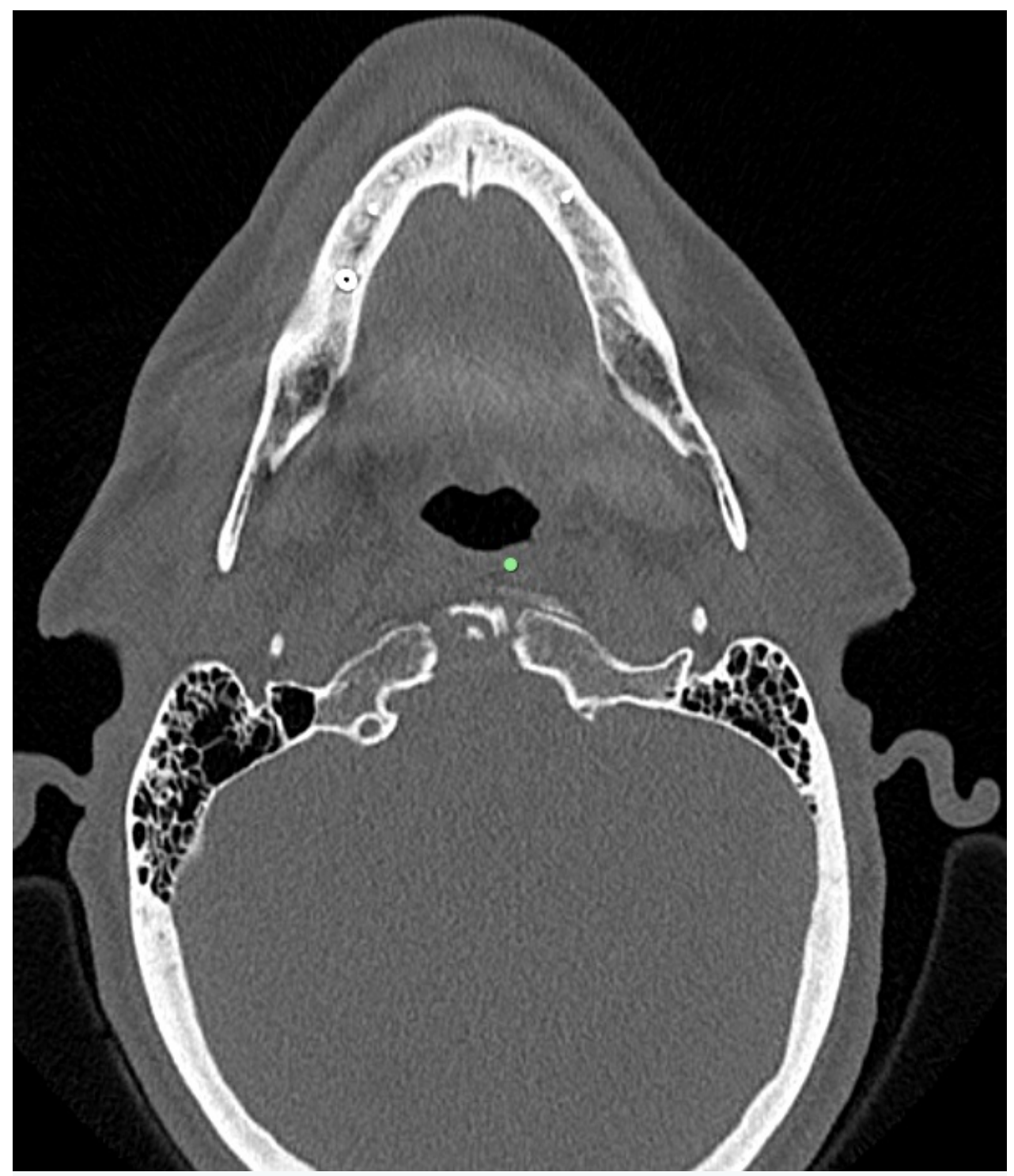

CT imagine at May 2015

Final diagnosis was of ONJ stage $2 \mathrm{~b}$ according to Italian recommendations and staging system ${ }^{[7][8]}$. He was treated with "conservative" treatment (antibiotic therapy) without severe infective episodes in the next three years.

In January 2018 bone exposure appeared at oral cavity ispection and a new CT scan showed increase of mandible alterations (Fif 3). 


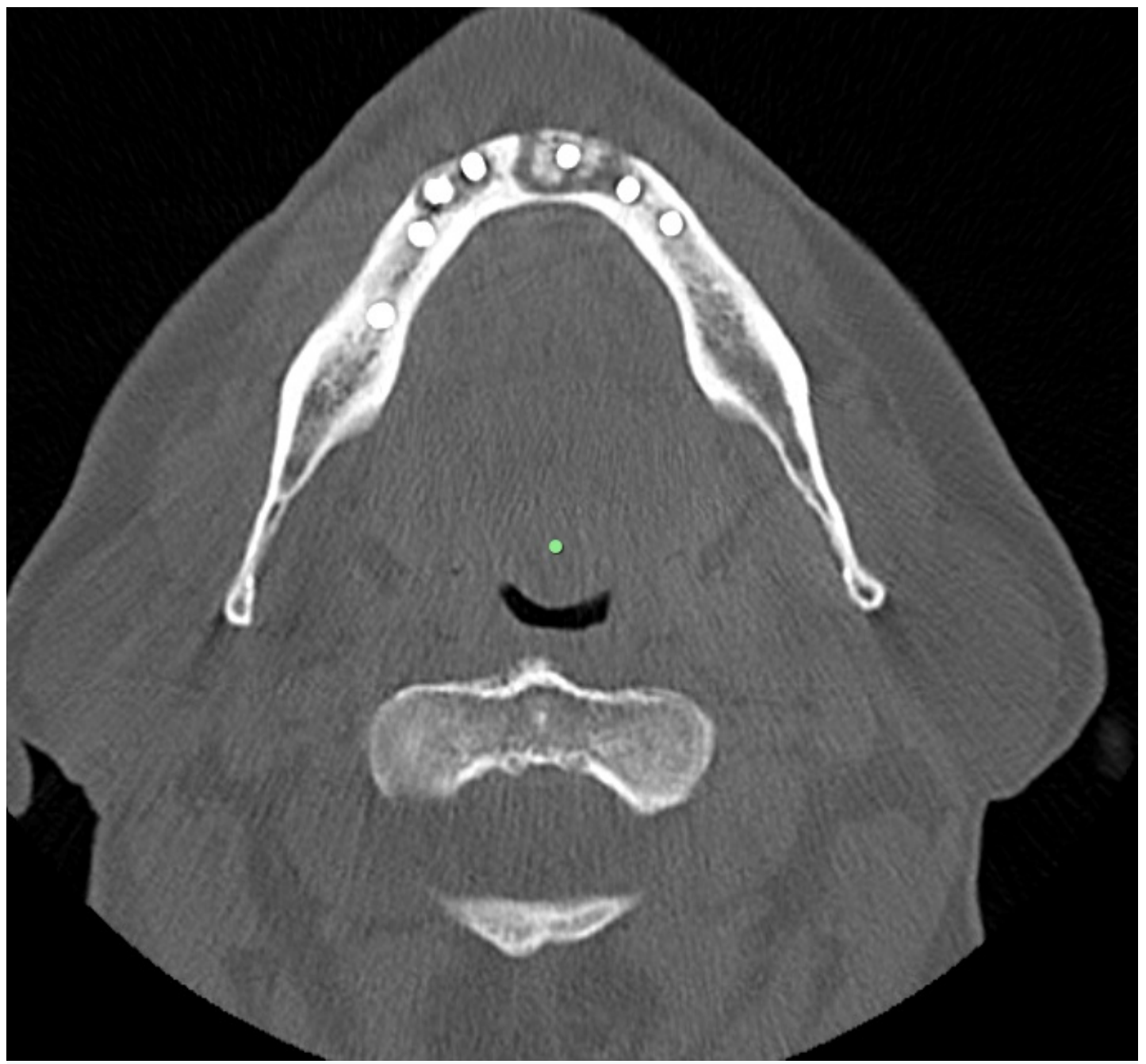

CT imagine at January 2018

A fragment of bone sequestrum was removed from the left hemi-mandible proximal region in April 2018. An improvement of oral condition was observed.

The patient is alive at 13 years from the start of antiresorptive treatment and at 5 years from the ONJ diagnosis.

\section{Conclusions:}

In advanced iodine-refractory DTC lenvatinib is standard of care. In patients with BM, bisphosphonates are highly effective in prevent SREs. The concomitant use of antiresorptive drugs (especially if administered for years) and antiangiogenic therapies is potentially associated with an increased risk of ONJ. Patients who undergo these treatment strategies must be carefully monitored to early recognize and prevent this rare but severe complication.

\section{References}

1. 'A. Nervo, A. Ragni, F. Retta, M. Gallo, et al. (2020). Bone metastases from differentiated thyroid carcinoma: current 
knowledge and open issues. J Endocrinol Invest, vol. 44 (3), 403-419. doi:10.1007/s40618-020-01374-7.

2. ^Nicole M. Iñiguez-Ariza, Keith C. Bible, Bart L. Clarke. (2020). Bone metastases in thyroid cancer. Journal of Bone Oncology, vol. 21 , 100282. doi:10.1016/j.jbo.2020.100282.

3. ^Yorihisa Orita, Iwao Sugitani, Kazuhisa Toda, Jun Manabe, et al. (2011). Zoledronic Acid in the Treatment of Bone Metastases from Differentiated Thyroid Carcinoma. Thyroid, vol. 21 (1), 31-35. doi:10.1089/thy.2010.0169.

4. ^Yorihisa Orita, Iwao Sugitani, Soshi Takao, Kazuhisa Toda, et al. (2015). Prospective Evaluation of Zoledronic Acid in the Treatment of Bone Metastases from Differentiated Thyroid Carcinoma. Ann Surg Oncol, vol. 22 (12), $4008-4013$. doi:10.1245/s10434-015-4497-0.

5. ^Martin Schlumberger et al. (2015). Lenvatinib versus placebo in radioiodine-refractory thyroid cancer. N Engl J Med. 2015 Feb 12;372(7):621-30. doi: 10.1056/NEJMoa1406470..

6. ^R. Mauceri, V. Panzarella, I. Morreale, G. Campisi. (2019). Medication-related osteonecrosis of the jaw in a cancer patient receiving lenvatinib. International Journal of Oral and Maxillofacial Surgery, vol. 48 (12), 1530-1532. doi:10.1016/j.ijom.2019.07.010.

7. ^A Bedogni, V Fusco, A Agrillo, G Campisi. (2012). Learning from experience. Proposal of a refined definition and staging system for bisphosphonate-related osteonecrosis of the jaw (BRONJ). Oral Diseases, vol. 18 (6), $621-623$. doi:10.1111/j.1601-0825.2012.01903.x.

8. ^Alberto Bedogni, Giuseppina Campisi, Vittorio Fusco, Alessandro Agrillo. (2013). Raccomandazioni clinicoterapeutiche sull'osteonecrosi delle ossa mascellari associata a bisfosfonati e sua prevenzione. Cleup. 